\section{DISTÚRBIOS OSTEOMUSCULARES AUTORREFERIDOS EM FUNCIONÁRIOS DE SUPERMERCADO}

\author{
Musculoskeletal disorders self-reported by supermarket \\ employees
}

\author{
Distúrbios osteomusculares auto reportados por empleados de \\ centro comercial
}

Artigo Original

\section{RESUMO}

Objetivo: Verificar a prevalência das algias autorreferidas em funcionários de uma rede de supermercados. Métodos: Estudo observacional, transversal, descritivo, realizado em uma rede de supermercados da capital paulista, no período de janeiro de 2011 a fevereiro de 2012, em uma amostra de 300 funcionários. Foram coletadas informações sociodemográficas, de atividade física e caracterização do processo de trabalho. Assumiu-se como desfecho o relato de sintomas de dores osteomusculares, por meio do Questionário Nórdico de Sintomas Osteomusculares. Para análise estatística, foram calculadas frequências e porcentagens. Resultados: A população, em sua maioria, era do sexo feminino, jovem, solteira e possuía escolaridade até o $2^{\circ}$ grau. Apenas $25 \%$ dos funcionários realizavam atividades físicas. Todos haviam apresentado algum tipo de sintomatologia musculoesquelética nos últimos 12 meses e metade $(50 \%)$ apresentou três ou mais sintomas. A dor apareceu predominante nos membros inferiores, seguida pela coluna torácica e lombar. A idade pode estar associada ao aparecimento de dores no pescoço. Além disso, a função associa-se a dores no cotovelo, coluna lombar e membros inferiores. Por fim, a coluna lombar é a região com maior associação dentre as variáveis independentes. Conclusão: Identificou-se que os funcionários investigados da referida rede de supermercado apresentavam prevalência de algias ou algum sintoma musculoesquelético nos últimos 12 meses, que englobaram o membro inferior, regiões que compõem a coluna vertebral, região dos punhos, dedos e mãos.

Descritores: Transtornos Traumáticos Cumulativos; Saúde do Trabalhador; Doenças Profissionais.

\section{ABSTRAC}

Objective: To determine the prevalence of self-reported pains in employees of a supermarket chain. Methods: A descriptive cross-sectional observational study conducted in a supermarket chain in the city of São Paulo from January 2011 to February 2012, with a sample of 300 employees. Information on sociodemographics, physical activity and characterization of the labor process were collected. It was assumed as the outcome the reports of symptoms of musculoskeletal pain obtained through The Nordic Musculoskeletal Questionnaire. For statistical analysis, frequencies and percentages were calculated. Results: The population was mostly composed of young, single women who attended up to the 2nd year of high school. Only $25 \%$ of employees performed physical activities. All employees had presented some type of musculoskeletal symptoms in the last 12 months, and half of them (50\%) had three or more symptoms. The pain predominantly occurred in the lower limbs, followed by the thoracic and lumbar spine. Age may be associated with the onset of neck pain. In addition, the job is associated with pain in elbows, lumbar spine and legs. Finally, the lumbar spine is the region with the highest association among the independent variables. Conclusion: It was verified that the employees investigated in the supermarket chain presented a prevalence of pains or some type of musculoskeletal symptom in the past 12 months in the lower limb, regions that make up the spine, wrists, fingers and hands.

Descriptors: Cumulative Trauma Disorders; Occupational Health; Occupational Diseases.

\author{
Bruna Almeida M. da Silva ${ }^{(1)}$ \\ Renata Nunes da Silva ${ }^{(1)}$ \\ Fernando Pereira Carlos ${ }^{(1)}$ \\ Luanda A. Collange Grecco ${ }^{(1)}$ \\ Leandro Henrique Grecco ${ }^{(2)}$
}

1) Universidade Nove de Julho - UNINOVE - São Paulo (SP) - Brasil

2) Universidade de São Paulo - USP - São Paulo (SP) - Brasil
Recebido em: 23/02/2013 Revisado em: $31 / 10 / 2013$ Aceito em: 12/12/2013 


\section{RESUMEN}

Objetivo: Verificar la prevalencia de dolores auto reportadas por empleados de una red de centros comerciales. Métodos: Estudio observacional, transversal y descriptivo, realizado en una red de centros comerciales en una capital entre enero de 2011 y febrero de 2012 con una muestra de 300 empleados. Fueron recogidas informaciones sociodemográficas, de actividad física y caracterización del proceso de trabajo. Se asumió como desenlace el relato de síntomas de dolores osteomusculares a través del Cuestionario Nórdico de Sintomas Osteomusculares. En el análisis estadístico fueron calculados frecuencias $y$ porcentajes. Resultados: La población era en su mayoría del sexo femenino, joven, soltera y de educación secundaria como máxima escolaridad. Solamente el $25 \%$ de los empleados realizaban actividades físicas. Todos habian presentado algún tipo de sintomatología en la musculatura esquelética de los últimos 12 meses y la mitad (50\%) presentó tres o más sintomas. El dolor fue predominante en los miembros inferiores, seguido de la columna torácica y lumbar. La edad puede estar asociada a la aparición de dolores del cuello. Además, la función se asocia a dolores del codo, columna lumbar y miembros inferiores. Por fin, la columna lumbar es la región con más asociación de las variables independientes. Conclusión: Se identificó que los empleados investigados de la referida red de centro comercial presentaron prevalencia de dolores o algún síntoma musculoesquelético en los últimos 12 meses que incluyeron el miembro inferior, regiones que constituyen la columna vertebral, puños, dedos y manos.

Descriptores: Trastornos de Trauma Acumulados; Salud Laboral; Enfermedades Profesionales.

\section{INTRODUÇÃO}

Os sintomas osteomusculares relacionados ao trabalho atingem diversas categorias profissionais e possuem várias denominações, entre as quais, lesões por esforços repetitivos (LER) e distúrbios osteomusculares relacionados ao trabalho (DORT), adotadas pelos Ministérios da Saúde e da Previdência Social ${ }^{(1)}$. Correspondem a um conjunto de afecções relacionadas às atividades laborativas, acometendo músculos, fáscias musculares, tendões, ligamentos, articulações, nervos, vasos sanguíneos e tegumento ${ }^{(2)}$. As várias formas clínicas de manifestação das LER/DORT têm como aspecto comum a dor e as incapacidades funcionais temporárias ou permanentes ${ }^{(3)}$.

Suas causas são múltiplas e complexas, originadas de fatores isolados e em conjuntos, mas que exercem seus efeitos de forma simultânea e interligada ${ }^{(4)}$. Fatores como o manuseio e o transporte de cargas realizadas de forma inapropriada; as posturas críticas, como rotação e flexão de tronco, e as posturas estáticas (prolongadas e mantidas); e a frequência (repetitividade dos mesmos movimentos) durante as atividades de trabalho têm sido postuladas com as principais causa do aparecimento de $\mathrm{LER} \mathrm{DORT}{ }^{(5)}$. Esses fatores ergonômicos são pela ocorrência dos sintomas musculoesqueléticos ${ }^{(6)}$.

Conhecida como a doença da modernidade, tem causado inúmeros afastamentos do trabalho, cuja quase totalidade evolui para incapacidade parcial e, em muitos casos, para incapacidade permanente, com aposentadoria por invalidez ${ }^{(7)}$. Em 2011, foram gastos no Brasil R\$ 356.038.000,00 com 381.810 auxílios-doença concedidos para trabalhadores com doenças do sistema osteomuscular, sendo a segunda maior concessão de auxílio-doença. Também é a segunda maior causa de aposentadoria por invalidez, com 23.485 aposentadorias no ano de 2011, que equivalem a um valor de $\mathrm{R} \$ 24.073 .000,00^{(8)}$.

As doenças osteomusculares surgem quando os limites físicos, fisiológicos e psicológicos dos trabalhadores são ultrapassados $^{(9)}$. Os doentes com LER/DORT apresentam queixa de dor, parestesias, sensação de peso e fadiga nos membros superiores e região cervical, geralmente com instalação insidiosa. Não raramente, apresentam também dor na região dorso-lombar e nos membros inferiores. Outros sintomas incluem adormecimento, alterações neurovegetativas, tróficas e outras anormalidades sensitivas e motoras regionais ${ }^{(3)}$, além de transtornos psicológicos e isolamento físico. É nesse momento que se torna significativo o apoio social, de familiares, profissionais de saúde, amigos, colegas e vizinhos no auxílio da recuperação e de enfrentamento da doença ${ }^{(4,10)}$.

As principais ações propostas no campo da prevenção e controle das doenças ocupacionais levam em conta a possibilidade da promoção da saúde no ambiente de trabalho, determinando as condições de risco, a caracterização e a quantificação dos fatores de risco, atingindo as empresas, os órgãos públicos e o mercado informal ${ }^{(11)}$. As intervenções preventivas realizadas atualmente trabalham com um grupo de atividades centradas nos indivíduos, como a ginástica laboral, os exercícios de pausa compensatória, as correções posturais in loco e os treinamentos de manejo de peso. Ressalta-se a necessidade dos cuidados com o corpo não somente no trabalho, mas também fora dele ${ }^{(12)}$.

Dentre diversas categorias profissionais afetadas pelas doenças osteomusculares, estão os funcionários de diversos setores de um supermercado. A incidência de LER/ DORT, por exemplo, é comum nos operadores de caixas de supermercado (checkout). Nessa categoria profissional, a LER/DORT está relacionada, geralmente, à introdução de novas tecnologias, como a informática e a leitura óptica, sem a adaptação dos postos e do ritmo de trabalho à nova situação ${ }^{(13)}$. De acordo com o Bureau of Labor \& Statistics, em 2000, havia mais de 20 mil distúrbios osteomusculares em trabalhadores de supermercados nos Estados Unidos. Mais de 50\% dessas lesões musculoesqueléticas estavam 
relacionadas ao levantamento de materiais nas lojas (repositores) $^{(14)}$.

Sabe-se da importância de estudos transversais que descrevam o que ocorre com um determinado grupo e em um determinado momento, e que eles são importantes guias para tomadas de decisões no setor de planejamento de saúde, influenciando o raciocínio clínico e a tomada de decisões nas práticas clinicas ${ }^{(15)}$. Portanto, o objetivo do presente estudo foi verificar a prevalência das algias autorreferidas em funcionários de uma rede de supermercados.

\section{MÉTODOS}

A presente investigação é um estudo observacional transversal descritivo realizado em uma rede de supermercados (4 lojas) da capital paulista, no período de janeiro de 2011 a fevereiro de 2012.

$\mathrm{O}$ convite aos funcionários para participar da pesquisa ocorreu respeitando sua disponibilidade. A opção de participar ou não surgiu após uma rápida explicação sobre o estudo. Destacou-se a não obrigatoriedade da participação, além de se garantir o sigilo das informações.

A amostra de conveniência investigou 300 funcionários de diversos setores do supermercado, sendo incluídos aqueles funcionários com mais de 1 ano no estabelecimento e aqueles sem nenhum diagnóstico prévio de problemas osteomusculares, e excluídos os funcionários que estavam em licença saúde ou outro tipo de afastamento durante o período da coleta de dados.

Além das informações sociodemográficas (sexo, idade e escolaridade) e da caracterização do processo de trabalho no supermercado (função, jornada e tempo de serviço), aqui consideradas como variáveis independentes, foram incluídas questões sobre atividades físicas, como tipo e frequência.

Assumiu-se como desfecho o relato de sintomas de dores osteomusculares, medido por meio do Questionário Nórdico de Sintomas Osteomusculares (QNSO), versão traduzida e validada para o português ${ }^{(16)}$. O instrumento avalia sintomas de dor no pescoço, ombro, cotovelo, antebraço, punho/mão/dedo, região dorsal, região lombar, quadril/coxa, joelho, tornozelo/pé, abordando dados pessoais, profissionais e sintomatologia musculoesquelética relacionados ao trabalho nos últimos doze meses e suas consequências. Aplicou-se o instrumento de coleta de dados por meio de entrevista autoaplicada em uma sala reservada no próprio local de trabalho. Não houve tempo estabelecido para o preenchimento, e os pesquisadores estavam presentes no local para esclarecimento de quaisquer dúvidas.

Para análise estatística, utilizaram-se as frequências e porcentagens para as regiões acometidas, trabalhos realizados, dados demográficos e atividades físicas. Aplicaram-se os testes $\left(\mathrm{x}^{2}\right)$ e $\mathrm{V}$ de Cramer para possíveis associações das variáveis categóricas entre as regiões acometidas e sexo, faixa etária, função, horas de trabalho e tempo de trabalho. Considerou-se o nível de significância de $5 \%$. Os dados foram tabulados e tratados através do programa estatístico SPSS 19.0.

O estudo recebeu a aprovação do Comitê de Ética em Pesquisa da Universidade Nove de Julho (CoEPUNINOVE, sob o n ${ }^{\circ} 432376 / 2011$ ) e todos os entrevistados assinaram o Termo de Consentimento Livre e Esclarecido, conforme orientação da Resolução 196/96.

\section{RESULTADOS}

Participaram da presente pesquisa 300 funcionários de 4 estabelecimentos da rede de supermercado estudada, sendo a população inquirida, em sua maioria, feminina $(n=195 / 56 \%)$, jovem entre 23 e 27 anos $(n=105 / 35 \%)$ e solteira $(n=177 / 59 \%)$, possuindo escolaridade até o $2^{\circ}$ grau $(n=272 / 90,7 \%)$ (Tabela I).

Observou-se também a distribuição dos relatos de atividades físicas e a frequência de duração realizadas pelos funcionários fora do âmbito de trabalho. Apenas $25 \%$ $(n=75)$ dos funcionários responderam realizar atividades físicas regularmente, com no mínimo 30 minutos de duração. Entre os que relataram a prática de atividade física, a predominante foi o futebol $(n=28 / 37,4 \%)$, seguida de caminhada ( $n=19 / 25,6 \%)$.

A Tabela II mostra a distribuição dos colaboradores dos diversos setores do supermercado. $\mathrm{O}$ expediente de trabalho diário, na maioria dos casos, era de 8 horas ( $n=267 / 89 \%$ ), com tempo de exercício da função de 1 a 3 $\operatorname{anos}(\mathrm{n}=156 / 52 \%)$ (Tabela II).

Todos os participantes $(n=300 / 100 \%)$ apresentaram algum tipo de sintomatologia musculoesquelética nos últimos 12 meses e 50,3\% $(n=151)$ apresentaram três ou mais sintomas. Consideraram-se as respostas "frequentemente" e "sempre" como parâmetros para se definir a prevalência das algias autorreferidas, sendo a predominância da dor nos membros inferiores, seguida pela coluna torácica, lombar e ombros, as de maiores ocorrências (Tabela III).

As associações entre algias autorreferidas, dados sociodemográficos e caracterização do processo de trabalho estão representadas na Tabela IV. No presente estudo, verificou-se que a idade pode estar associada ao aparecimento de dores no pescoço, e que a função associa-se a dores no cotovelo, coluna lombar e membros inferiores. Por fim, foi observado que dores na coluna lombar representam a região com maior associação dentre as variáveis independentes realizadas no presente estudo. 
Tabela I - Caracterização sociodemográfica dos funcionários de uma rede de supermercado. São Paulo-SP, 2011-2012.

\begin{tabular}{|c|c|c|}
\hline Dados sociodemográficos & $\mathbf{n}$ & $\%$ \\
\hline \multicolumn{3}{|l|}{ Faixa etária } \\
\hline$<18$ anos & 2 & 0,7 \\
\hline 18 a 22 anos & 78 & 26,0 \\
\hline 23 a 27 anos & 105 & 35,0 \\
\hline 28 a 32 anos & 54 & 18,0 \\
\hline+32 anos & 61 & 20,3 \\
\hline \multicolumn{3}{|l|}{ Sexo } \\
\hline Feminino & 195 & 65,0 \\
\hline Masculino & 105 & 35,0 \\
\hline \multicolumn{3}{|l|}{ Estado civil } \\
\hline Casado & 123 & 41,0 \\
\hline Solteiro & 177 & 59,0 \\
\hline \multicolumn{3}{|l|}{ Escolaridade } \\
\hline Até $2^{\circ}$ grau & 272 & 90,7 \\
\hline Superior incompleto & 20 & 6,7 \\
\hline Superior completo & 8 & 2,7 \\
\hline
\end{tabular}

Tabela II - Distribuição dos funcionários segundo função, horas trabalhadas e tempo de serviço. São Paulo-SP, 2011-2012.

\begin{tabular}{lcc}
\hline Dados da função & n & \% \\
\hline Função & 66 & 22,0 \\
Caixa & 40 & 13,3 \\
Promotor & 22 & 7,3 \\
Balconista & 33 & 11,0 \\
Repositor & 31 & 10,3 \\
Vendedor & 1 & 0,3 \\
Analista de sistema & 14 & 4,7 \\
Fiscal & 4 & 1,3 \\
Motorista & 2 & 0,7 \\
Líder & 22 & 7,3 \\
Administrativo & 13 & 4,3 \\
Auxiliar de limpeza & 52 & 17,3 \\
Atendente & & \\
Horas de serviço & 5 & 1,7 \\
6 horas & 267 & 89,0 \\
8 horas & 28 & 9,3 \\
$\quad 8$ horas & & \\
Tempo de serviço & 51 & 17,0 \\
1 ano & 156 & 52,0 \\
1 a 3 anos & 61 & 20,3 \\
4 a 6 anos & 20 & 6,7 \\
7 a 10 anos & 12 & 4,0 \\
> 10 anos & &
\end{tabular}


Tabela III - Frequência de sintomas autorreferidos por região anatômica de funcionários de uma rede de supermercados. São Paulo-SP, 2011-2012.

\begin{tabular}{lcccccccc}
\hline \multirow{2}{*}{ Região corporal } & \multicolumn{2}{c}{ Nenhuma } & \multicolumn{2}{c}{ Raramente } & \multicolumn{2}{c}{ Frequentemente } & \multicolumn{2}{c}{ Sempre } \\
& $\mathbf{n}$ & $\mathbf{0}$ & $\mathbf{n}$ & $\mathbf{\%}$ & $\mathbf{n}$ & $\mathbf{\%}$ & $\mathbf{n}$ & $\mathbf{\%}$ \\
\hline Pescoço & 157 & 52,3 & 107 & 35,7 & 19 & 6,3 & 17 & 5,7 \\
Ombro & 122 & 40,7 & 75 & 25 & 51 & 17 & 52 & 17,3 \\
Braço & 133 & 44,3 & 64 & 21,3 & 62 & 20,7 & 41 & 13,7 \\
Cotovelo & 282 & 94 & 16 & 5,3 & 2 & 0,7 & 0 & 0 \\
Antebraço & 201 & 67 & 54 & 18 & 21 & 7 & 24 & 8 \\
P. M. D. & 140 & 46,7 & 43 & 14,3 & 53 & 17,7 & 64 & 21,3 \\
Coluna torácica & 50 & 16,7 & 86 & 28,7 & 81 & 27 & 83 & 27,7 \\
Coluna lombar & 67 & 22,3 & 76 & 25,3 & 76 & 25,3 & 81 & 27 \\
Membros inferiores & 58 & 19,3 & 57 & 19 & 90 & 30 & 95 & 31,7 \\
\hline
\end{tabular}

P.M.D: Punhos, Mãos e Dedos

Tabela IV - Possíveis associações das variáveis categóricas entre as regiões acometidas e sexo, faixa etária, função, horas de trabalho, e tempo de trabalho. São Paulo-SP, 2011-2012.

\begin{tabular}{|c|c|c|c|c|c|}
\hline Região corporal & Sexo & Idade & Função & H. trabalho & A. função \\
\hline Pescoço & 0,55 & $0,03 *$ & 0,39 & $<0,01 *$ & 0,07 \\
\hline Ombro & 0,32 & 0,27 & 0,11 & $0,05 *$ & 0,16 \\
\hline Braço & 0,47 & 0,81 & 0,62 & $0,01 *$ & 0,58 \\
\hline Cotovelo & 0,14 & 0,83 & $0,02 *$ & 0,25 & $0,03 *$ \\
\hline Antebraço & 0,06 & 0,97 & 0,40 & 0,69 & 0,42 \\
\hline P. M. D. ${ }^{*}$ & 0,58 & 0,58 & 0,18 & $0,04 *$ & $<0,01 *$ \\
\hline Coluna torácica & 0,14 & 0,14 & 0,57 & 0,16 & 0,30 \\
\hline Coluna lombar & 0,14 & 0,14 & $0,05^{*}$ & $<0,01^{*}$ & $0,03 *$ \\
\hline Membros inferiores & 0,72 & 0,72 & $0,05^{*}$ & 0,06 & 0,79 \\
\hline
\end{tabular}

H.trabalho: horas no trabalho; A.função: anos na função; P.M.D: Punhos, Mãos e Dedos. * $\mathrm{p} \leq 0,05$

\section{DISCUSSÃO}

Todos os funcionários $(\mathrm{n}=300 / 100 \%)$ da rede de supermercado estudada na atual investigação apresentaram algum sintoma álgico osteomuscular, percebido de forma autorreferida. Metade ( $\mathrm{n}=151 / 50,3 \%)$ apresentou três ou mais sintomas. Além disso, esses sintomas apresentaram associação com as seguintes variáveis: idade, horas de trabalho e tempo na função. Não se observou associação entre os sintomas autorreferidos e a não prática de atividades físicas.

O presente estudo demonstrou o perfil desses funcionários, verificando a prevalência de adultos jovens, solteiros e do sexo feminino. A maior parte dos trabalhadores do setor de comércio possui idade entre 18 e 24 anos. Muitos desses jovens têm no comércio o seu primeiro emprego, principalmente porque são pequenas ou inexistentes as exigências de conhecimentos técnicos e experiência anterior ${ }^{(17)}$. Esses resultados corroboram com um estudo no qual os autores observaram a prevalência de funcionários do sexo feminino, com idade média de 25 anos e $2^{\circ}$ grau completo ${ }^{(18)}$.

As LER/DORT estão fundamentalmente relacionadas com as mudanças na organização do trabalho e com as inovações tecnológicas resultantes da reestruturação produtiva. Essas transformações no processo produtivo vêm levando a uma maior intensificação do trabalho, com hipersolicitação de tendões, músculos e articulações dos trabalhadores ${ }^{(19)}$. Tradicionalmente, o trabalho do operador de caixa em supermercados tem sido analisado, no âmbito da ergonomia, sob o ponto de vista fisiológico e biomecânico. O resultado evidenciado no presente estudo foi que operadores de caixas representaram o maior grupo de funcionários entrevistados, podendo ser uma justificativa para o grande acervo de estudos relacionados a esse grupo de trabalhadores ${ }^{(6,20-22)}$.

Após a introdução da leitura óptica, houve um aumento de incidência de queixas relacionadas à fadiga muscular. Além disso, o operador de caixa sofre pressão para evitar 
a formação de grandes filas de espera, e cobrança por parte dos clientes por um serviço mais rápido, eficiente e perfeito. Esses fatores fazem com que o trabalhador acelere o ritmo de trabalho e, consequentemente, aumente a sobrecarga física e mental( ${ }^{(22)}$. Mas os caixas estão longe de serem os únicos trabalhadores de supermercado afetados por distúrbios musculoesqueléticos. As pesquisas ${ }^{(6,20-22)}$ realizadas na área apontam para isso, porém, mais estudos a fim de promover a saúde do trabalhador ou ao menos amenizar os problemas relativos aos funcionários de outros setores de um supermercado se fazem necessários.

Um estudo observou que cerca de um terço dos trabalhadores de uma rede de supermercados, de diversos setores, relatou algum tipo de desconforto, sendo as dores lombares uma das mais referidas ${ }^{(23)}$. Diferentemente, no presente estudo, metade desses trabalhadores apresentou um ou mais sintomas nos últimos 12 meses. Outros grandes estudos investigaram a prevalência de dores lombares em todos os funcionários de diversos setores do supermercado e demonstraram uma prevalência de $34 \%^{(24)}$ e $45 \%{ }^{(21)}$ da amostra global. Sugere-se existir uma relação potencial entre sintomas psicossomáticos relacionados ao estresse, embora não com a satisfação no trabalho ${ }^{(24)}$, ponto não analisado no estudo em questão, no qual essa porcentagem alcançou $50 \%$ dos funcionários.

A dor lombar está associada não apenas ao trabalhador que realiza atividades de grande esforço físico, como o levantamento e o transporte de cargas, mas também ao trabalhador exposto ao trauma de efeito cumulativo presente em trabalhos considerados leves ${ }^{(22)}$. Isso pode ser visto com os resultados aqui apresentados, pois o aparecimento da dor lombar se correlacionou não somente com a função exercida, mas também com as horas trabalhadas por dia e o tempo na função. Entre os fundamentais fatores de risco relacionados aos distúrbios musculoesqueléticos, estão a organização do trabalho, os fatores ambientais e as prováveis sobrecargas de segmentos corporais em determinados movimentos, como: força excessiva para realizar determinadas tarefas, repetitividade de movimentos e de posturas inadequadas no desenvolvimento das atividades laborais, e fatores fisiológicos ${ }^{(25)}$.

Embora se consiga obter uma história ocupacional detalhada, a relação entre a doença ou agravo à saúde e a ocupação/profissão exercida ficará clara se baseada na observação direta do trabalho do paciente ${ }^{(26)}$. No que se refere à jornada e ao tempo de trabalho, observou-se, na presente investigação, que a maioria $(\mathrm{n}=267 / 89 \%)$ trabalhava entre um e três anos, tendo uma jornada de 8 horas por dia. A informação da organização do trabalho (trabalho manual repetitivo, alta carga física, carga estática, ferramentas vibrantes, aumento da intensidade e duração da exposição) é considerada um dos fatores de risco mais comuns, principalmente quando acrescenta outros fatores, como: pausas curtas ou inexistentes, pressão temporal, exigência e incentivo à produtividade, ritmo e trabalho imposto pela gerência ou linha de montagem (esteiras), horas extras, trabalho monótono e sem conteúdo, sobrecarga de produção (picos de produção absenteísmo, falta de programação) e falta de treinamento ${ }^{(9,27,28)}$.

Buscou-se compreender a relação das regiões anatômicas onde predominam os sintomas e a frequência das manifestações de dor, provocadas pelo exercício profissional. Esses resultados concordam com estudos que descrevem as LER/DORT como doenças com sintomatologia global, e não apenas em membros superiores ${ }^{(5,29,30)}$. $O$ presente estudo constatou o predomínio de queixas na região dos membros inferiores, seguida pela coluna torácica e lombar, ombros e punhos. Apesar de o estudo não realizar uma avaliação física, esses achados sugerem um ressaltante impacto da postura nos sintomas osteomusculares. É sabido que uma postura prolongada pode ocasionar sobrecarga estática sobre as fibras musculares, originando dor e desconforto. Muitas vezes, essas estruturas estão associadas às condições do local de trabalho, favorecendo o surgimento de sintomas musculares ${ }^{(31)}$. Posturas inadequadas, concentração de movimento e utilização generalizada do computador podem favorecer o aparecimento de LER/DORT ${ }^{(32)}$.

Quanto à atividade física, apenas $25 \%$ dos entrevistados disseram praticá-la. A mecanização de várias atividades, aliada a uma intensa jornada de trabalho, está afastando a prática esportiva da vida do ser humano. O trabalhador que reserva alguns minutos da semana para exercitar-se terá mais disposição e seu serviço irá render ${ }^{(18)}$. Cabe ressaltar que a recomendação da American College Sports Medicine (ACSM) é realizar atividade física de 3 a 5 dias por semana, por pelo menos 30 minutos de duração ${ }^{(33)}$. Apesar desses dados, não houve associação entre os sintomas relacionados ao trabalho e a atividade física na presente investigação.

Os resultados da atual pesquisa têm importância para o direcionamento de iniciativas preventivas e de promoção da saúde dos trabalhadores da saúde de forma mais eficiente. No entanto, a questão de sua origem é um desafio a ser superado pelos conflitos e controvérsias existentes, e envolvem pesquisadores, profissionais da saúde e trabalhadores. Assim, a necessidade de políticas preventivas efetivas a partir dos diversos segmentos envolvidos com o trabalhador e com o trabalhar e suas múltiplas relações é reconhecidamente uma prioridade, sendo, portanto, a medida mais importante envolvendo esse fenômeno ${ }^{(27)}$. Dessa forma, a dimensão biomecânica deve ser pesquisada juntamente com a dimensão organizacional e a psicossocial. Por conseguinte, a pesquisa interdisciplinar pode ser uma solução para racionalizar os gastos e melhorar a transação de conhecimentos entre as áreas ${ }^{(34)}$. 
Não foram apresentados dados sobre a realização de atividades domésticas, juntamente com outros hábitos diários, que poderiam contribuir para o surgimento de sintomas musculoesqueléticos. $\mathrm{O}$ uso excessivo do computador no âmbito doméstico e as atividades domésticas com movimentos repetitivos (lavar e passar roupa, lavar louça e limpar vidros) podem agravar os quadros de dor osteomuscular, porém, não podem ser consideradas causas isoladas desses sintomas, por apresentarem flexibilidade de ritmo e tempo ${ }^{(28)}$.

A principal limitação do presente estudo foi a técnica de amostragem de conveniência, que pode ter afetado a generalização dos resultados. Uma possível avaliação clínico-funcional poderia ser realizada, a fim de correlacionar prováveis achados clínicos com os sintomas autorreferidos. A utilização de instrumentos de autopreenchimento pode tornar-se um viés, uma vez que é possível sofrer influência das funções cognitivas, da cultura, da linguagem e da escolaridade. Outra limitação aceitável refere-se à possibilidade de viés, pois os entrevistados respondiam sobre o estado que predominou no período de um ano anterior à entrevista, podendo não se lembrar de todos os incidentes de LER/DORT. O estudo não investigou a interrelação entre os distúrbios osteomusculares e os fatores de risco, tais como a carga física, a carga psicossocial e o estado geral de saúde.

\section{CONCLUSÃO}

Identificou-se que os funcionários investigados da referida rede de supermercado apresentaram prevalência de algias ou algum sintoma musculoesquelético nos últimos 12 meses, englobando membros inferiores, regiões que compõem a coluna vertebral, região dos punhos, dedos e mãos.

\section{REFERÊNCIAS}

1. Settimi MM, Almeida IM, Toledo LF, Paparelli R, Silva JÁ, Martins M. Lesões por Esforços Repetitivos (LER)/Distúrbios Osteomusculares relacionados ao trabalho (DORT). São Paulo: CEREST; 2000.

2. Al-Eisa E, Buragadda S, Shaheen AAM, Ibrahim A, Melam GR. Work related musculoskeletal disorders: causes, prevalence and response among egyptian and saudi physical therapists. Middle-East J. Sci. Res. 2012;12(4):523-9.

3. Yeng LT, Teixeira MJ, Romano MA, Picarelli H, Settimi MM, Greve JMA. Distúrbios ósteo-musculares relacionados ao trabalho. Rev Med. 2001;80(Ed. Esp. Pt.2):422-42.
4. Torres ARA, Chagas MIO, Moreira ACA, Barreto ICHC, Rodrigues EM. O adoecimento no trabalhador: repercurssões na vida do trabalhador e de sua família. Sanare. 2011;10(1):42-8.

5. Anap DB, Iyer C, Rao K. Work related musculoskeletal disorders among hospital nurses in rural Maharashtra, India: a multi centre survey. Int $J$ Res Med Sci. 2013;1(2):101-7.

6. Draicchio F, Trebbi M, Mari S, Forzano F, Serrao M, Sicklinger A, et al. Biomechanical evaluation of supermarket cashiers before and after a redesign of the checkout counter. Ergonomics. 2012;55(6):650-69.

7. Rosa AFG, Garcia PA, Vedoato T, Campos RG, Lopes MLS. Incidência de LER/DORT em trabalhadores de enfermagem. Acta Sci. 2008;30(1):19-25.

8. Empresa de Tecnologia e Informações da Previdência Social - DATAPREV. Anuário Estatístico da Previdência Social 2011. Brasília: Ministério da Previdência; 2011.

9. Moraes PWT, Bastos AVB. As LER/DORT e os fatores psicossociais. Arq Bras Psicol. 2013;65(1):2-20.

10. Saldanha JHS, Pereira APM, Neves RF, Lima MAG. Facilitadores e barreiras de retorno ao trabalho de trabalhadores acometidos por LER/DORT. Rev Bras Saúde Ocup. 2013;38(127):122-38.

11. Picoloto D, Silveira ED. Prevalência de sintomas osteomusculares e fatores associados em trabalhadores de uma indústria metalúrgica de Canoas-RS. Ciênc Saúde Coletiva. 2008;13(2):507-16.

12. Delani D, Evangelista RA, Pinho ST, Silva AC. Ginástica laboral: melhoria na qualidade de vida do trabalhador. Rev Cient Faema. 2013;4(1):41-61.

13. Riesco TB, Sandoval RA, Kappes V. Exercícios compensatórios laborais nos operadores de" checkout" de um supermercado de Goiânia. Lect Educación Fís Deportes. 2006;10(92):26.

14. Clarke CM. Workplace injuries and illnesses in grocery stores. Compensation and Working Conditions. Washington: Department of Labor, Bureau of Labor Statistics; 2003. p.1-12.

15. Curci KA, Oliveira MR, Souza Rangel MM, Mendes S. Promoção da saúde e prevenção de riscos e doenças na Saúde Suplementar: um breve histórico. Mundo Saúde. 2013;37(2):230-40.

16. Pinheiro FA, Tróccoli BT, Carvalho CV. Validação do questionário nórdico de sintomas osteomusculares 
como medida de morbidade. Rev Saúde Pública. 2002;36(3):307-12.

17. Matos LB. Jovens e adolescentes no mercado de trabalho: uma análise sobre o Programa de Aprendizagem e suas Implicações nas empresas do polo de Manaus. Rev Magistro. 2013;8(2):77-90.

18. Battisti HH, Guimarães ACA, Simas JPN. Atividade física e qualidade de vida de operadores de caixa de supermercado. Rev Bras Ciência Mov. 2005;13(1):718.

19. Murofuse NT, Marziale MHP. Doenças do sistema osteomuscular em trabalhadores de enfermagem. Rev Latinoam Enferm. 2005;13(3):364-73.

20. Bonfiglioli R, Mattioli S, Fiorentini C, Graziosi F, Curti S, Violante FS. Relationship between repetitive work and the prevalence of carpal tunnel syndrome in part-time and full-time female supermarket cashiers: a quasi-experimental study. Int Arch Occup Environ Health. 2007;80(3):248-53.

21. Forcier L, Lapointe C, Lortie M, Buckle P, Kuorinka I, Lemaire J, et al. Supermarket workers: Their work and their health, particularly their self-reported musculoskeletal problems and compensable injuries. Work. 2008;30(4):493-510.

22. Trelha CS, Cunha ACV, Carregaro RL, Castro RFD, Gallo JMCL, Silva DW, et al. Prevalência de sintomatologia musculoesquelética em funcionários de supermercado em Lonsdrina-PR; Musculoskeletal symptoms in workers of supermarket of Londrina-PR. Fisioter Mov. 2004;17(4):59-64.

23. Ryan GA. The prevalence of musculo-skeletal symptoms in supermarket workers. Ergonomics. 1989;32(4):359-71.

24. Violante FS, Graziosi F, Bonfiglioli R, Curti S, Mattioli S. Relations between occupational, psychosocial and individual factors and three different categories of back disorder among supermarket workers. Int Arch Occup Environ Health. 2005;78(8):613-24.

25. Magnago TSBS, Lisboa MTL, Griep RH, Kirchhof ALC, Camponogara S, Nonnenmacher CQ, et al. Nursing workers: work conditions, social-demographic characteristics and skeletal muscle disturbances. Acta Paul Enferm. 2010;23(2):187-93.

26. Galvão JT. Saúde e qualidade de vida do operador de caixa de supermercado. Palmas. Monografia [Bacharelado em Administração] - Universidade de Brasília; 2012.
27. Karino ME, Martins JT, Bobroff MCC. Reflexão sobre as políticas de saúde do trabalhador no brasil: avanços e desafios. Ciênc Cuid Saúde. 2011;10(2):395-400.

28. Martins AC, Felli VEA. Sintomas músculoesqueléticos em graduandos de enfermagem. Enferm Foco. 2013;4(1):58-62.

29. Etemadinezhad S, Ranjbar F, Gorji M. Posture analysis by OWAS method and prevalence of Musculoskeletal Disorders using Nordic questionnaire among workers of Sourak tobacco factory in 2013. IJEHSE. 2013;1(2):89-94.

30. Moraes PWT, Bastos AVB. As LER/DORT e os fatores psicossociais. Arq Bras Psicol. 2013;65(1):2-20.

31. Mergener CR, Kehrig RT, Traebert J. Sintomatologia músculo-esquelética relacionada ao trabalho e sua relação com qualidade de vida em bancários do Meio Oeste Catarinense. Saúde Soc. 2008;17(4):171-81.

32. Antunes ED, Araújo CR, Abage Z. Musculoskeletal sympotms in workers of a Telecom Company. Work. 2012;41(Suppl. 1):5725-7.

33. American College of Sports Medicine. ACSM's exercise management for persons with chronic diseases and disabilities. Indianapolis: Human Kinetics Publishers; 2003.

34. Silva EP, Minette LJ, Souza AP, Marçal MA, Sanches ALP. Psychosocial and organizational factors associated with risk of LER/DORT in operators of forest harvesting machines. Rev Árvore. 2013;37(5):889-95.

\section{Endereço do primeiro autor:}

Bruna Almeida M. da Silva

Universidade Nove de Julho (UNINOVE)

Avenida Dr. Adolpho Pinto, 109

Bairro: Santa Cecília

CEP: 01156-050 - São Paulo - SP - Brasil

E-mail: almeida.fisio@yahoo.com.br

\section{Endereço para correspondência:}

Leandro Henrique Grecco

Universidade de São Paulo (USP)

Departamento de Farmacologia

Avenida Professor Lineu Prestes, 1524

Bairro: Cidade Universitária

CEP: 05508-900 - São Paulo - SP - Brasil

E-mail: leandrohgrecco@usp.br 